\title{
On the equivalence between the energy and virial routes to the equation of state of hard-sphere fluids
}

\author{
Andrés Santo:* \\ Departamento de Física, Universidad de Extremadura, E-06071 Badajoz, Spain
}

(Dated: October 7, 2013)

\begin{abstract}
The energy route to the equation of state of hard-sphere fluids is ill-defined since the internal energy is just that of an ideal gas and thus it is independent of density. It is shown that this ambiguity can be avoided by considering a square-shoulder interaction and taking the limit of vanishing shoulder width. The resulting hard-sphere equation of state coincides exactly with the one obtained through the virial route. Therefore, the energy and virial routes to the equation of state of hard-sphere fluids can be considered as equivalent.
\end{abstract}

PACS numbers:

Given a fluid of particles interacting via a two-body potential $\varphi(r)$, its equation of state (EOS) can be obtained in terms of the radial distribution function $g(r ; \rho, \beta)$, where $\rho$ is the number density and $\beta=1 / k_{B} T$ is the inverse temperature, through a number of routes $\frac{1}{n}$ The most common ones are the virial route,

$\frac{\beta p}{\rho} \equiv Z(\rho, \beta)=1+2^{d-1} v_{d} \rho \int_{0}^{\infty} \mathrm{d} r r^{d} y(r ; \rho, \beta) \frac{\partial}{\partial r} e^{-\beta \varphi(r)}$,

the compressibility route

$$
\begin{aligned}
\left(\beta \frac{\partial p}{\partial \rho}\right)^{-1} \equiv \chi(\rho, \beta)= & 1+2^{d} d v_{d} \rho \int_{0}^{\infty} \mathrm{d} r r^{d-1} \\
& \times[h(r ; \rho, \beta)-1],
\end{aligned}
$$

and the energy route

$$
\begin{aligned}
u(\rho, \beta)= & \frac{d}{2 \beta}\left[1+2^{d} v_{d} \rho \beta \int_{0}^{\infty} \mathrm{d} r r^{d-1} \varphi(r) e^{-\beta \varphi(r)}\right. \\
& \times y(r ; \rho, \beta)] .
\end{aligned}
$$

In Eqs. (11)-(3), $p$ is the pressure, $Z$ is the compressibility factor, $d$ is the dimensionality of the system, $v_{d}=(\pi / 4)^{d / 2} / \Gamma(1+d / 2)$ is the volume of a $d$-dimensional sphere of unit diameter, $\chi$ is the isothermal susceptibility, $u$ is the internal energy per particle, $y(r) \equiv$ $\exp [\beta \varphi(r)] g(r)$ is the cavity function, and $h(r) \equiv g(r)-1$ is the total correlation function. If the exact function $g(r ; \rho, \beta)$ is inserted, the three routes are thermodynamically consistent, i.e.,

$$
\begin{gathered}
\chi^{-1}(\rho, \beta)=\frac{\partial}{\partial \rho}[\rho Z(\rho, \beta)], \\
\rho \frac{\partial}{\partial \rho} u(\rho, \beta)=\frac{\partial}{\partial \beta} Z(\rho, \beta) .
\end{gathered}
$$

On the other hand, if an approximate function $g(r ; \rho, \beta)$ is used, the compressibility factor obtained directly from Eq. (11) does not necessarily coincide with that obtained from the combination of Eqs. (2) and (4) or with that obtained from the combination of Eqs. (3) and (5).
Let us now particularize to the hard-sphere (HS) interaction

$$
\varphi_{\mathrm{HS}}(r)= \begin{cases}\infty, & r<\sigma, \\ 0, & r>\sigma .\end{cases}
$$

In that case, $y(r ; \rho, \beta)=y_{\mathrm{HS}}\left(r ; \rho \sigma^{d}\right)$ and Eq. (1) becomes

$$
Z_{\mathrm{HS}}\left(\rho \sigma^{d}\right)=1+2^{d-1} v_{d} \rho \sigma^{d} y_{\mathrm{HS}}\left(\sigma ; \rho \sigma^{d}\right) .
$$

On the other hand, Eq. (3) reduces to

$$
u_{\mathrm{HS}}(\beta)=\frac{d}{2 \beta} .
$$

Since $Z_{\mathrm{HS}}$ is independent of temperature and $u_{\mathrm{HS}}$ is independent of density, Eq. (5) is trivially satisfied as $0=0$ and so it is not possible in principle to get the compressibility factor from the internal energy.

A way of circumventing the ill-definition of the energy route to the EOS of an HS fluid consists of considering a convenient interaction potential that encompasses that of hard spheres in certain limits but for which the temperature plays a relevant role. The simplest choice for such a potential is perhaps the so-called square-shoulder (SS) potential:2

$$
\varphi_{\mathrm{SS}}(r)= \begin{cases}\infty, & r<\sigma \\ \epsilon, & \sigma<r<\sigma^{\prime}, \\ 0, & r>\sigma^{\prime},\end{cases}
$$

where $\epsilon$ is a positive constant. For this potential, Eq. (31) becomes

$u_{\mathrm{SS}}(\rho, \beta)=\frac{d}{2 \beta}\left[1+2^{d} v_{d} \rho \beta \epsilon e^{-\beta \epsilon} \int_{\sigma}^{\sigma^{\prime}} \mathrm{d} r r^{d-1} y_{\mathrm{SS}}(r ; \rho, \beta)\right]$.

The SS interaction is equivalent to an HS interaction of diameter $\sigma$ in the infinite-temperature limit $(\beta \epsilon \rightarrow 0)$ and to an HS interaction of diameter $\sigma^{\prime}$ in the zerotemperature limit $(\beta \epsilon \rightarrow \infty)$. Of course, the equivalence $\mathrm{SS} \rightarrow \mathrm{HS}$ also holds in the limit of zero shoulder width $\left(\sigma^{\prime} \rightarrow \sigma\right)$. 
Let us now imagine that $y_{\mathrm{SS}}(r ; \rho, \beta)$ is known, either exactly or approximately (e.g., as obtained from the Percus-Yevick approximation), and thus it is possible to compute $u_{\mathrm{SS}}(\rho, \beta)$ from Eq. (10). Then, we can get $Z_{\mathrm{SS}}(\rho, \beta)$ from Eq. (5) as

$$
\begin{aligned}
Z_{\mathrm{SS}}(\rho, \beta)-Z_{\mathrm{HS}}\left(\rho \sigma^{d}\right)= & 2^{d-1} d v_{d} \epsilon \rho \frac{\partial}{\partial \rho} \rho \int_{0}^{\beta} \mathrm{d} \beta^{\prime} e^{-\beta^{\prime} \epsilon} \\
& \times \int_{\sigma}^{\sigma^{\prime}} \mathrm{d} r r^{d-1} y_{\mathrm{SS}}\left(r ; \rho, \beta^{\prime}\right),
\end{aligned}
$$

where we have made use of the property $\lim _{\beta \epsilon \rightarrow 0} Z_{\mathrm{SS}}(\rho, \beta)=Z_{\mathrm{HS}}\left(\rho \sigma^{d}\right)$. Taking into account the complementary condition $\lim _{\beta \epsilon \rightarrow \infty} Z_{\mathrm{SS}}(\rho, \beta)=$ $Z_{\mathrm{HS}}\left(\rho{\sigma^{\prime}}^{d}\right)$, Eq. (111) yields

$$
\begin{aligned}
\frac{Z_{\mathrm{HS}}\left(\rho{\sigma^{\prime}}^{d}\right)-Z_{\mathrm{HS}}\left(\rho \sigma^{d}\right)}{\rho{\sigma^{\prime}}^{d}-\rho \sigma^{d}}= & \frac{2^{d-1} d v_{d} \epsilon}{{\sigma^{\prime}}^{d}-\sigma^{d}} \frac{\partial}{\partial \rho} \rho \int_{0}^{\infty} \mathrm{d} \beta e^{-\beta \epsilon} \\
& \times \int_{\sigma}^{\sigma^{\prime}} \mathrm{d} r r^{d-1} y_{\mathrm{SS}}(r ; \rho, \beta) .
\end{aligned}
$$

Equation (12) can be considered as the condition defining the compressibility factor of an HS fluid associated with the energy route. To proceed further, we take the limit $\sigma^{\prime} \rightarrow \sigma$. Thus,

$$
\begin{aligned}
& \lim _{\sigma^{\prime} \rightarrow \sigma} \frac{Z_{\mathrm{HS}}\left(\rho{\sigma^{\prime}}^{d}\right)-Z_{\mathrm{HS}}\left(\rho \sigma^{d}\right)}{\rho \sigma^{\prime d}-\rho \sigma^{d}}=\sigma^{-d} \frac{\partial}{\partial \rho} Z_{\mathrm{HS}}\left(\rho \sigma^{d}\right), \\
& \lim _{\sigma^{\prime} \rightarrow \sigma} \frac{1}{{\sigma^{\prime}}^{d}-\sigma^{d}} \int_{\sigma}^{\sigma^{\prime}} \mathrm{d} r r^{d-1} y_{\mathrm{SS}}(r ; \rho, \beta)=\frac{1}{d} y_{\mathrm{HS}}\left(\sigma ; \rho \sigma^{d}\right) .
\end{aligned}
$$

Therefore, Eq. (12) reduces in the limit $\sigma^{\prime} \rightarrow \sigma$ to

$$
\frac{\partial}{\partial \rho} Z_{\mathrm{HS}}\left(\rho \sigma^{d}\right)=2^{d-1} v_{d} \frac{\partial}{\partial \rho} \rho \sigma^{d} y_{\mathrm{HS}}\left(\sigma ; \rho \sigma^{d}\right) .
$$

Finally, integrating over density and imposing the ideal gas boundary condition $Z_{\mathrm{HS}}(0)=1$, the virial EOS (7) is reobtained.

The generalization to mixtures (either additive or nonadditive) is straightforward. In that case, the SS potential for each pair $i j$ is defined by Eq. (9) with the changes $\epsilon \rightarrow \epsilon_{i j}, \sigma \rightarrow \sigma_{i j}$, and $\sigma^{\prime} \rightarrow \sigma_{i j}^{\prime}=\lambda \sigma_{i j}$, where the factor $\lambda$ is common to all the pairs. The equation equivalent to Eq. (12) in the case of mixtures is

$$
\begin{array}{r}
\frac{Z_{\mathrm{HS}}\left(\rho{\sigma_{\text {eff }}^{\prime}}^{d}\right)-Z_{\mathrm{HS}}\left(\rho \sigma_{\text {eff }}^{d}\right)}{\rho\left(\lambda^{d}-1\right)}=\frac{2^{d-1} d v_{d}}{\lambda^{d}-1} \frac{\partial}{\partial \rho} \rho \sum_{i j} x_{i} x_{j} \\
\times \epsilon_{i j} \int_{0}^{\infty} \mathrm{d} \beta e^{-\beta \epsilon_{i j}} \int_{\sigma_{i j}}^{\sigma_{i j}^{\prime}} \mathrm{d} r r^{d-1} y_{i j}^{\mathrm{SS}}(r ; \rho, \beta),
\end{array}
$$

where $\left\{x_{i}\right\}$ are mole fractions and $\sigma_{\text {eff }}^{d} \equiv \sum_{i, j} x_{i} x_{j} \sigma_{i j}^{d}$, ${\sigma^{\prime}}_{\text {eff }}^{d} \equiv \sum_{i, j} x_{i} x_{j}{\sigma^{\prime}}_{i j}^{d}=\lambda^{d} \sigma_{\text {eff }}^{d}$. Taking the limit $\lambda \rightarrow 1$ in Eq. (16) one gets

$$
Z_{\mathrm{HS}}\left(\rho \sigma_{\mathrm{eff}}^{d}\right)=1+2^{d-1} v_{d} \rho \sum_{i j} x_{i} x_{j} \sigma_{i j}^{d} y_{i j}^{\mathrm{HS}}\left(\sigma_{i j} ; \rho \sigma_{\mathrm{eff}}^{d}\right),
$$

which is not but the virial EOS for an HS mixture.

In summary, in this note I have shown that the illdefinition of the energy route to the EOS of an HS fluid is saved by first considering an SS fluid and then taking the limit of vanishing shoulder width. The resulting EOS coincides exactly with the one obtained through the virial route. From that point of view, the energy and virial routes to the EOS of HS fluids can be considered as equivalent.

\section{Acknowledgments}

This research has been supported by the Ministerio de Educación y Ciencia (Spain) through Grant No. FIS2004-01399 (partially financed by FEDER funds).
* Electronic address: andres@unex.es URL: http://www. unex.es/eweb/fisteor/andres/

1 J.-P. Hansen, I.R. McDonald, Theory of Simple Liquids, Academic Press, London, 1986.

2 D. M. Heyes and P. J. Aston, J. Chem. Phys. 97, 5738 (1992); C. Rascón, E. Velasco, L. Mederos, and G. Navascués, ibid. 106, 6689 (1997); A. R. Denton and H. Löwen, J. Phys.: Condens. Matt. 9, L1 (1997); P. Bolhuis and D. Frenkel, ibid. 9, 381 (1997); A. Lang, G. Kahl, C. N. Likos, H. Löwen, and M. Watzlawek, ibid. 11, 10143 (1999); A. L. Benavides and A. Gil-Villegas, Mol. Phys. 97, 1225 (1999); A. Vidales, A. L. Benavides, and A. Gil-Villegas, ibid. 99, 703 (2001); A. J. Schultz, C. K. Hall, and J. Genzer, J. Chem. Phys. 117, 10329 (2002); G. Malescio and G. Pellicane, Nature Materials 2, 97 (2003). 